Check for updates

Cite this: RSC Adv., 2019, 9, 16439

\title{
A glucose biosensor based on the immobilization of glucose oxidase and Au nanocomposites with polynorepinephrine
}

\begin{abstract}
Yang Liu, $\uparrow$ Xu Nan, $\uparrow$ Wei Shi, (D) * Xin Liu, Zi He, Yanan Sun and Dongtao Ge*
A glucose biosensor based on Au nanoparticles (AuNPs), glucose oxidase (GOD) and polynorepinephrine (PNE) was fabricated by a simple and green approach. PNE-functionalized AuNPs (AuNPs(APNE) were synthetized by the polymerization of norepinephrine (NE) onto the surfaces of AuNPs and casted on an $\mathrm{Au}$ electrode. After dropping a solution containing NE and GOD on the AuNPs@PNE-modified Au electrode and oxidizing the monomer NE by cyclic voltammetry, a PNE, GOD and AuNP-modified Au electrode (PNE/GOD/AuNPs(aPNE/Au) was obtained. The biosensor presented high sensitivity of $35.4 \mu \mathrm{A}$ $\mathrm{mM}^{-1} \mathrm{~cm}^{-2}$ to glucose in the range from $0.003 \mathrm{mM}$ to $3.43 \mathrm{mM}$ with a response time of less than $3 \mathrm{~s}$, a detection limit of $1.34 \mu \mathrm{M}$ at a signal/noise ratio of 3, a low Michaelis-Menten constant (6.8 $\mathrm{mM})$ and outstanding selectivity as well as stability. Moreover, the sensitivity and linear detection range of the asprepared biosensor were further enhanced by the addition of the redox mediator $p$-benzoquinone.
\end{abstract}

Received 17th March 2019

Accepted 12th May 2019

DOI: $10.1039 / c 9 r a 02054 c$

rsc.li/rsc-advances

AuNPs. ${ }^{1}$ Therefore, new strategies for developing electrochemical biosensors with extraordinary electrochemical activity and stability are still urgently needed.

Inspired by mussels in nature, Lee et al. found that polydapamine (PDA) is a good adhesion material for surface modification. ${ }^{9}$ Besides, the favorable conductivity and abundant amino groups of PDA for conjugating biometric probes make it quite suitable for the electrode modification of electrochemical biosensors. Thus, PDA-based electrochemical biosensors are superior to majority of biopolymers (such as chitosan) and exhibit better sensitivity along with improved stability and working life. However, the rough surface structure of PDA makes the distance between the active center of the enzyme and the electrode variable, which might be unfavorable for efficient electron transport. Recently, polynorepinephrine (PNE), an analogue of PDA, was found to be more apt to form homogeneous and flattened wrap layers on a variety of materials owing to the large amount of dihydroxybenzaldehyde (DHBA) in its molecular structure. ${ }^{10,11}$ In addition, the abundant amino and hydroxyl groups of PNE are beneficial for conjugating various biometric probes. Thus, more stable and sensitive electrochemical biosensors could be obtained via simple PNE surface modification. However, to the best of our knowledge, PNE-based glucose oxidase electrochemical biosensors have not been reported yet.

In this paper, a high-performance enzyme electrode based on AuNPs, PNE and GOD was developed for glucose detection by a green method. To fabricate the enzyme electrode, AuNPs were coated with PNE via the polymerization of norepinephrine (NE) under alkaline conditions, followed by dropping AuNPs@PNE onto the surface of the Au electrode. Then, the solution

Key Laboratory of Biomedical Engineering of Fujian Province University, Research Center of Biomedical Engineering of Xiamen, Department of Biomaterials, College of Materials, Xiamen University, Xiamen 361005, China. E-mail: shiwei@xmu.edu.cn; gedt@xmu.edu.cn

$\dagger$ These authors contributed equally to this work. 
containing NE and GOD was dropped on the AuNPs@PNEmodified $\mathrm{Au}$ electrode. After oxidizing the monomer $\mathrm{NE}$ by cyclic voltammetry, the PNE film, which immobilized and protected the nanoparticles and GOD, was obtained and coated onto the electrode, as shown in Scheme 1. The as-prepared enzyme electrode combined the advantages of PNE (favorable adhesion, protectability and biocompatibility), AuNPs (high conductivity and amplification of the response current), and GOD (high selectivity and activity toward glucose); it also exhibited good sensitivity, a wide detection range, a low Michaelis constant, and excellent selectivity and antiinterference ability.

\section{Materials and methods}

\section{Reagents and instruments}

DL-Noradrenaline hydrochloride (NE) was purchased from Toronto Research Chemicals Inc (TRC, Canada). $\mathrm{HAuCl}_{4}$ was purchased from Shanghai Tuosi Chemical Co., Ltd (China). $\mathrm{C}_{6} \mathrm{H}_{5} \mathrm{Na}_{3} \mathrm{O}_{7}, \mathrm{H}_{2} \mathrm{SO}_{4}(98 \%), \mathrm{H}_{2} \mathrm{O}_{2}(30 \%), \mathrm{K}_{2} \mathrm{SO}_{4}, \mathrm{~K}_{2} \mathrm{HPO}_{4} \cdot 3 \mathrm{H}_{2} \mathrm{O}$, $\mathrm{KH}_{2} \mathrm{PO}_{4}, \mathrm{~K}_{3} \mathrm{Fe}(\mathrm{CN})_{6}, \mathrm{~K}_{4} \mathrm{Fe}(\mathrm{CN})_{6} \cdot 3 \mathrm{H}_{2} \mathrm{O}$, glucose and $\mathrm{KNO}_{3}$ were purchased from Xilong Scientific Co., Ltd (China). $\mathrm{C}_{6} \mathrm{H}_{5} \mathrm{Na}_{3} \mathrm{O}_{7}$, $\mathrm{KCl}$, ethanol and acetone were purchased from Shantou Dahao Fine Chemical Co., Ltd (China). Uric acid (UA), ascorbic acid (AA) and Tris base were purchased from Sinopharm Chemical Reagent Co., Ltd (China). Ultra-pure water $(18 \mathrm{M} \Omega \mathrm{cm})$ was obtained from Pen-Tung Sah MEMS Research Center of Xiamen University. $\mathrm{N}_{2}$ was purchased from Xiamen Kong Fen Te Qi Industry Co., Ltd (China).

A JEM-1400 transmission electron microscope (TEM, JOEL, Japan) was used to observe the morphology of the nanoparticles. Mastersizer 2000 (Mastersizer, UK), UV1750 (Shimadzu, Japan) and Nicolet iS10 (Thermo Fisher Scientific, China) were applied for the characterization of the nanoparticles. Cyclic voltammetry (CV) curves, electrochemical impedance spectroscopy (EIS) curves, and amperometric $i-t$ curves of the prepared electrodes were obtained with a CHI660D electrochemical workstation ( $\mathrm{CH}$ Instruments, USA). All of the electrochemical experiments were run in a three-electrode system composed of a modified electrode as the working electrode, a platinum wire as the auxiliary electrode, and a saturated calomel electrode (SCE) as the reference electrode. The CV tests

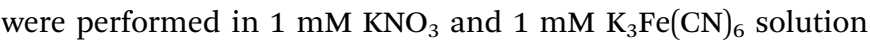
under a scan range of -0.2 to $0.6 \mathrm{~V}$ at $0.05 \mathrm{~V} \mathrm{~s}^{-1}$ at room temperature. The EIS measurements were carried out in $0.1 \mathrm{M}$ $\mathrm{KCl}$ and $5 \mathrm{mM} \mathrm{K}_{3} \mathrm{Fe}(\mathrm{CN})_{6} / \mathrm{K}_{4} \mathrm{Fe}(\mathrm{CN})_{6}$ solutions under the frequency range of $10 \mathrm{kHz}$ to $0.01 \mathrm{~Hz}$ with an amplitude of $5 \mathrm{mV}$. Amperometric curves were tested by adding glucose into the 0.1 M PBS solution ( $\mathrm{pH}$ 7.4) under constant agitation at an appropriate potential.

\section{Preparation of AuNPs@PNE}

$\mathrm{Au}$ nanoparticles (AuNPs) were prepared by reducing $\mathrm{HAuCl}_{4}$ with sodium citrate. ${ }^{\mathbf{1 2}}$ Briefly, $90 \mathrm{~mL}$ trisodium citrate solution (0.82 $\mathrm{mM}$ ) was heated up to $100{ }^{\circ} \mathrm{C}$ under reflux with rapid magnetic stirring. After the solution was boiling for $15 \mathrm{~min}$, $10 \mathrm{~mL} \mathrm{HAuCl}_{4}$ solution $(3.73 \mathrm{mM}$ ) was added. When the color of the reaction solution turned to claret-red, AuNPs were obtained and then stored at $4{ }^{\circ} \mathrm{C}$.

The prepared AuNP solution (10 mL) was mixed with $10 \mathrm{~mL}$ NE Tris solution ( $\mathrm{pH} 8.5,1 \mathrm{mg} \mathrm{mL}{ }^{-1}$ ) with mechanical stirring $(480 \mathrm{rpm})$ at room temperature for $1 \mathrm{~h}$. After centrifuging at $9000 \mathrm{rpm}$ for $30 \mathrm{~min}$, the precipitate was collected and dispersed in $1 \mathrm{~mL}$ ultra-pure water by ultrasonication. The obtained AuNPs@PNE nanoparticle solution was stored at $4{ }^{\circ} \mathrm{C}$.

\section{Preparation of the PNE/GOD/AuNPs@PNE/Au electrode}

An $\mathrm{Au}$ electrode was immersed in Piranha solution (70\% concentrated $\mathrm{H}_{2} \mathrm{SO}_{4}$ and $30 \% \mathrm{H}_{2} \mathrm{O}_{2}$ ) for $5 \mathrm{~min}$ to remove
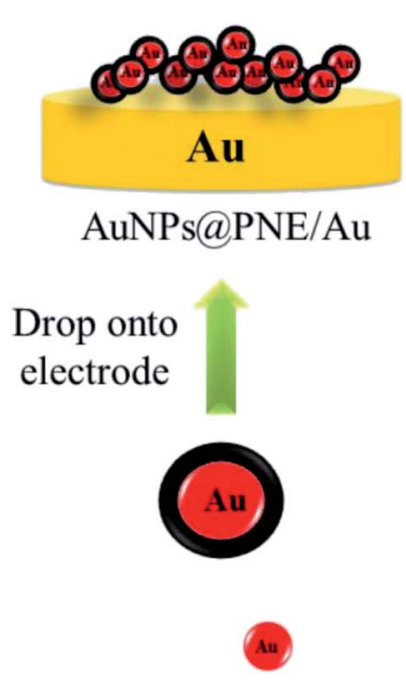

AuNPs
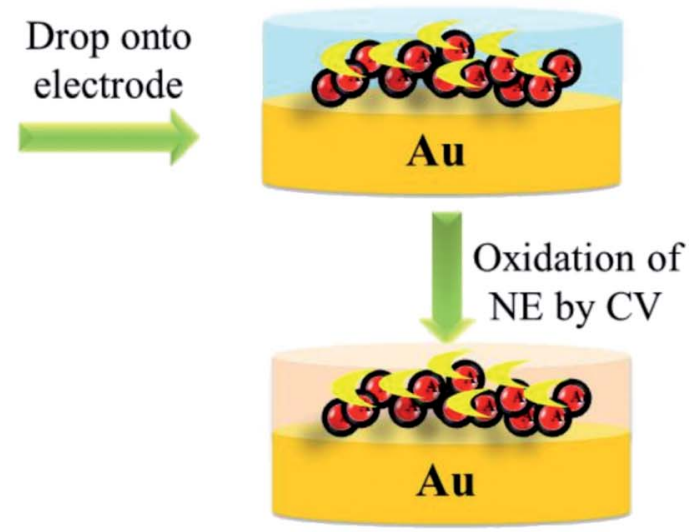

PNE/GOD/AuNPs@PNE/Au

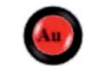

AuNPs@PNE

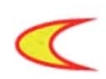

Glucose oxidase

Scheme 1 Schematic illustration of the fabrication of the PNE/GOD/AuNPs@PNE/Au electrode. 
impurities, followed by sonicating in deionized water for $5 \mathrm{~min}$. Subsequently, the Au electrode was polished by 1, 0.3, and 0.05 $\mu \mathrm{m} \mathrm{Al}{ }_{2} \mathrm{O}_{3}$ particles, washed with acetone, ethanol and deionized water by sonication, and then stored at $4{ }^{\circ} \mathrm{C}$.

To prepare the PNE/GOD/AuNPs@PNE/Au electrode, $8 \mu \mathrm{L}$ AuNPs@PNE solution was dropped onto the polished Au electrode, followed by drying at room temperature in vacuum. Then, $20 \mathrm{mg}$ NE and $20 \mathrm{mg}$ GOD were added to a $4 \mathrm{~mL}$ Tris solution (pH 8.5). Next, $8 \mu \mathrm{L}$ of the above solution was dropped onto the AuNPs@PNE modified electrode and dried at room temperature. The modified electrode (NE/GOD/AuNPs@PNE/Au) was oxidized by $\mathrm{CV}$ scan in PBS ( $\mathrm{pH}$ 7.4) under a scanning range of -0.5 to $0.5 \mathrm{~V}$ at $0.05 \mathrm{~V} \mathrm{~s}^{-1} .^{13}$ The obtained $\mathrm{PNE} / \mathrm{GOD} /$ AuNPs@PNE/Au electrode was stored at $4{ }^{\circ} \mathrm{C}$ for further use. For comparison, the $\mathrm{PNE} / \mathrm{GOD} / \mathrm{Au}$ electrode was prepared in a similar way without dropping AuNPs@PNE.

\section{Glucose determination in human blood serum samples}

Human blood serum samples were provided by the Zhongshan Hospital of Xiamen University (Xiamen, China). The samples $(500 \mu \mathrm{L})$ were added into $4 \mathrm{~mL} 0.1 \mathrm{M}$ PBS (pH 7.4). The current responses of the solution were measured at a potential of $0.7 \mathrm{~V}$ using the PNE/GOD/AuNPs@PNE/Au electrode as the working electrode. The glucose concentration of the samples was calculated from the working curve.

\section{Results and discussion}

\section{Characterization of AuNPs and AuNPs@PNE}

The surface morphologies of the as-prepared AuNPs and AuNPs@PNE were characterized by TEM. As shown in Fig. 1a, AuNPs have a smooth surface and homogeneous diameter $(\sim 10$ $\mathrm{nm}$ ). Due to outstanding biocompatibility and high adhesion, PNE was chosen to modify AuNPs by polymerizing NE onto the surface of AuNPs. The AuNPs were successfully coated by the PNE film and the mean diameter of the nanoparticles was about $20 \mathrm{~nm}$ (Fig. 1b). Dynamic light scattering (DLS) analysis (Fig. 1c) revealed that the hydrodynamic diameter ranges of AuNPs and AuNPs@PNE were 8-25 nm (peak at $11 \mathrm{~nm}$ ) and 10-40 nm (peak at $20 \mathrm{~nm}$ ), respectively, which were in agreement with the results of the TEM images. Subsequently, we obtained the UVvis absorption spectra of AuNPs@PNE (Fig. 1d). Compared to the observations for AuNPs, a new absorption peak for AuNPs@PNE at $280 \mathrm{~nm}$ was ascribed to PNE, further indicating that PNE was successfully coated on AuNPs. ${ }^{14}$

\section{Characterization of the electrodes}

Fig. 2a shows the CV scanning of the NE/GOD/AuNPs@PNE/Au electrode in PBS ( $\mathrm{pH}$ 7.4). It can be clearly seen that the current of the first cycle achieves a peak value $(58 \mu \mathrm{A})$ between 0.4 and 0.5 V. During CV scanning, the anodic peak decreased gradually and disappeared in the 10th cycle, indicating that NE was oxidized successfully and the electrochemical oxidation of $\mathrm{NE}$ was irreversible. To further verify the successful fabrication of the PNE/GOD/AuNPs@PNE/Au electrode, attenuated total reflectance-Fourier-transform infrared (ATR-FTIR) spectra of the AuNPs@PNE/Au electrode, NE/GOD/AuNPs@PNE/Au electrode and PNE/GOD/AuNPs@PNE/Au electrode were obtained for structural characterization (Fig. 2b). The AuNPs@PNE/Au electrode exhibited PNE FTIR characteristic peaks of the $\mathrm{C}=\mathrm{C}$ resonance vibrations of the benzene ring, the $\mathrm{N}-\mathrm{H}$ bending vibrations of amine at $1590 \mathrm{~cm}^{-1}$ and $1510 \mathrm{~cm}^{-1}$, and the $\mathrm{C}-\mathrm{N}$ stretching vibrations of amine and arylamine at $1144 \mathrm{~cm}^{-1}$ and $1200 \mathrm{~cm}^{-1}$ in the fingerprint region. ${ }^{15,16}$ The peaks at $1144 \mathrm{~cm}^{-1}$ and $1200 \mathrm{~cm}^{-1}$ disappeared in the spectrum of the NE/GOD/ AuNPs@PNE/Au electrode and the peaks at $1637 \mathrm{~cm}^{-1}$ and $1518 \mathrm{~cm}^{-1}$ appeared due to the coverage of GOD protein molecules and the typical amide bond absorption of GOD. ${ }^{17,18}$ After oxidizing NE by CV scanning of the NE/GOD/AuNPs@PNE/ $\mathrm{Au}$ electrode, the peaks at $1144 \mathrm{~cm}^{-1}$ and $1200 \mathrm{~cm}^{-1}$ appeared again and the peaks at $1637 \mathrm{~cm}^{-1}$ and $1518 \mathrm{~cm}^{-1}$ were still observed, confirming that NE was successfully polymerized into PNE and GOD was immobilized in the PNE film.

For calculating the diffusion coefficient, the redox reaction of the PNE/GOD/AuNPs@PNE/Au electrode was measured in a solution of $1 \mathrm{mM} \mathrm{K}_{3} \mathrm{Fe}(\mathrm{CN})_{6}$ and $0.1 \mathrm{M} \mathrm{KNO}_{3}$ by $\mathrm{CV}$ scans at different rates of 60, 80, 100, 200, 300, 400, 500, 600, 700, 800, 900 and $1000 \mathrm{mV} \mathrm{s}^{-1}$ with $\mathrm{Fe}(\mathrm{CN})_{6}{ }^{3-/ 4-}$ as the redox probe; moreover, the standard curve was made between electric current peak values and the square root of scan rates, as shown in Fig. 2c. Obviously, on increasing the scan rate, the electric current of the anodic peak shifted in the positive direction and increased, and there was a linearly positive correlation between the current values and the square root of scanning rates. The linear equation is $I_{\mathrm{P}}=0.20863 v^{1 / 2}-0.13168$ with $R=0.9987$. Similarly, as the scanning rate increased, the electric current of the cathodic peak shifted to the negative direction and increased, and there was a linearly negative correlation between the current values and the square root of scanning rates. The linear equation is $I_{\mathrm{P}}=-0.2946 v^{1 / 2}+0.42134$ with $R=0.9981$. The results indicated that the redox reaction of $\mathrm{Fe}(\mathrm{CN})_{6}{ }^{3-/ 4-}$ on the surface of the PNE/GOD/AuNPs@PNE/Au electrode is a diffusion-controlled process. The diffusion coefficient of the PNE/GOD/AuNPs@PNE/Au electrode was $5.54 \times 10^{-7} \mathrm{~cm}^{2} \mathrm{~s}^{-1}$ according to the Randles-Sevdik equation: ${ }^{19} i_{\mathrm{p}}=2.69 \times 10^{5} n^{3 /}$ ${ }^{2} A D^{1 / 2} C v^{1 / 2}$, where $A, D, i_{\mathrm{p}}, n, C$, and $v^{1 / 2}$ are the effective reaction area of the electrode, the diffusion coefficient, the current peak value, the number of electrons in the redox reaction, the concentration of $\mathrm{Fe}(\mathrm{CN})_{6}{ }^{3-/ 4-}$ and the square root of scanning rate, respectively.

Subsequently, electrochemical impedance spectroscopy (EIS) was applied for characterizing the interface properties of different electrodes. ${ }^{20}$ The Nyquist plots of the bare $\mathrm{Au}$ electrode, PNE/GOD/Au electrode, and PNE/GOD/AuNPs@PNE/Au electrode were obtained in solutions of $5 \mathrm{mM} \mathrm{K}_{3} \mathrm{Fe}(\mathrm{CN})_{6} /$ $\mathrm{K}_{4} \mathrm{Fe}(\mathrm{CN})_{6}$ and $0.1 \mathrm{M} \mathrm{KCl}$ with $\mathrm{Fe}(\mathrm{CN})_{6}{ }^{3-/ 4-}$ as the redox probe, as shown in Fig. $2 \mathrm{~d}$. The charge transfer resistance $\left(R_{\mathrm{ct}}\right)$, which is equal to the radius of the semicircle in the Nyquist plots, reflects the electron transfer kinetics of $\mathrm{Fe}(\mathrm{CN})_{6}{ }^{3-/ 4-}$ at the surface of the electrodes. The $R_{\mathrm{ct}}$ values of the bare Au electrode, PNE/GOD/Au electrode and PNE/GOD/AuNPs@PNE/Au electrode were 704.7, 4774 and $16190 \Omega$, respectively. The modified electrodes exhibited larger $R_{\mathrm{ct}}$ values than the bare Au electrode, 
a

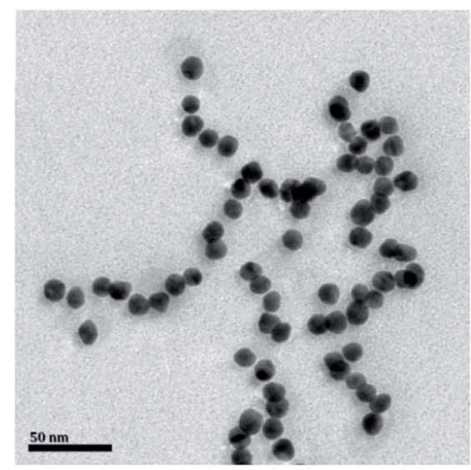

b

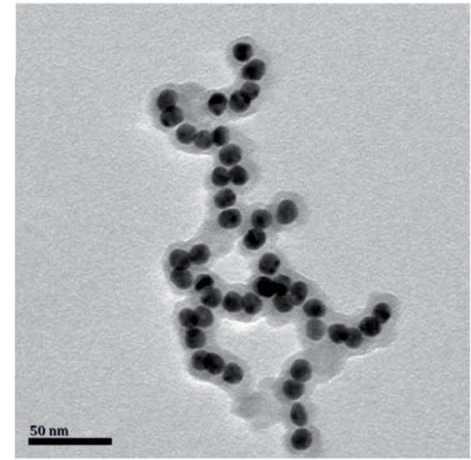

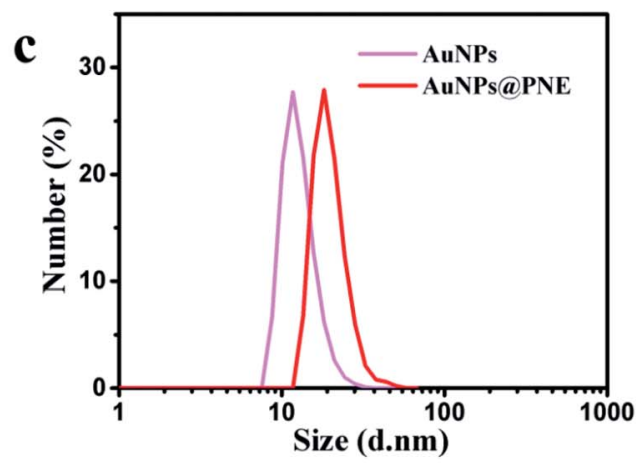

d

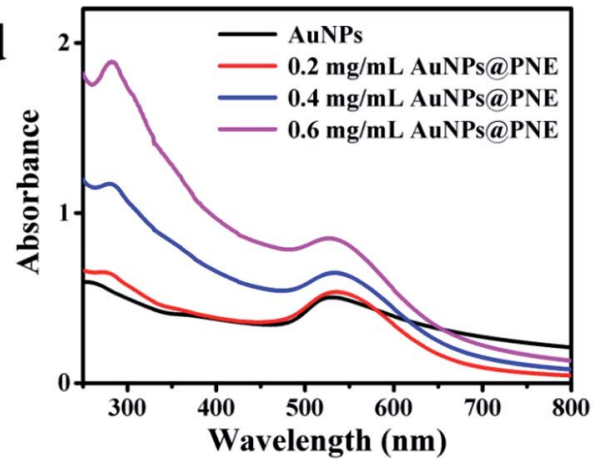

Fig. 1 (a, b) TEM images of AuNPs and AuNPs@PNE; (c) DLS analyses of AuNPs and AuNPs@PNE; (d) UV-vis absorbance spectra of AuNPs and AuNPs@PNE in water.
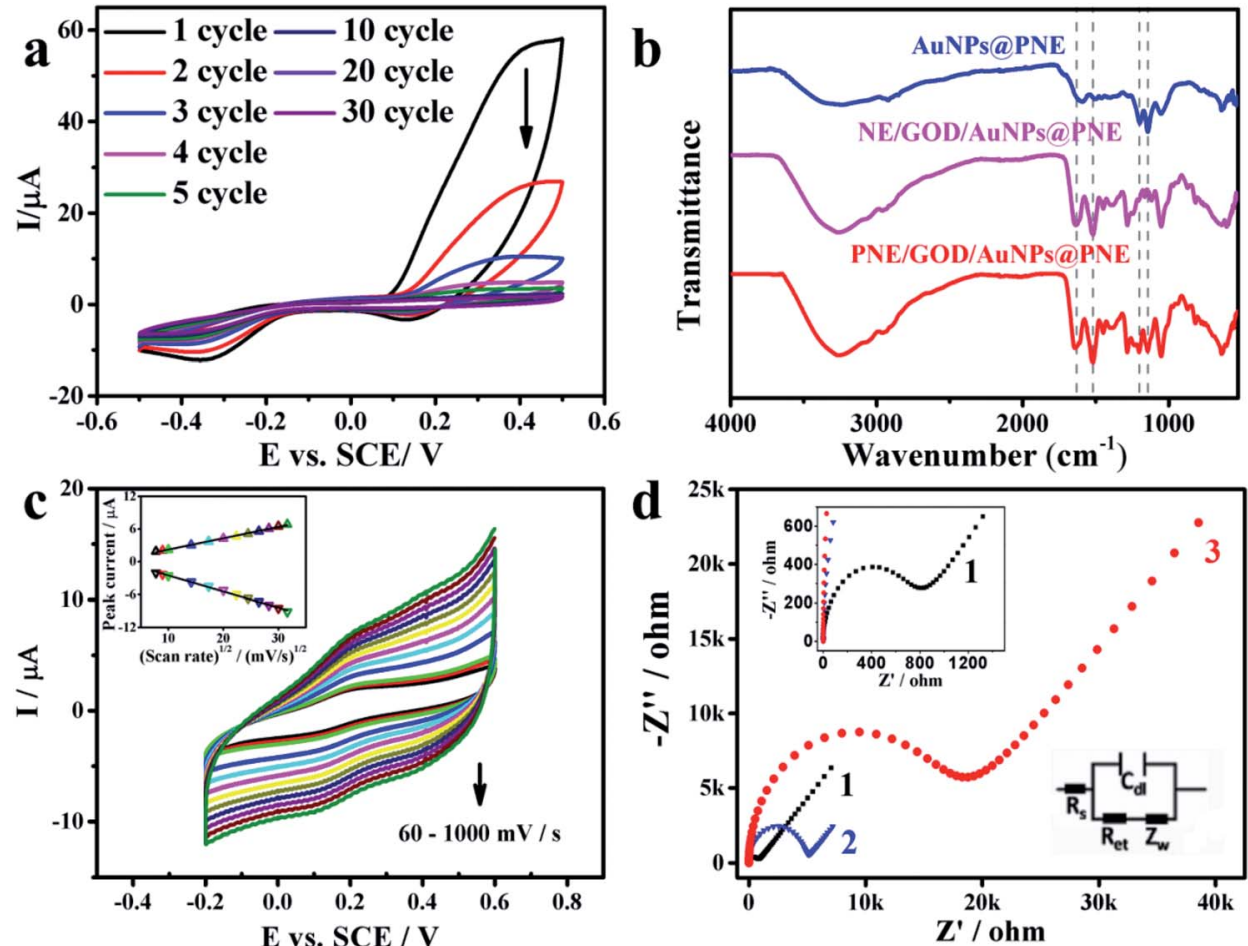

Fig. 2 (a) CV curves of the NE/GOD/AuNPs@PNE/Au electrode in PBS ( $\mathrm{pH} 7.4$ ) at $50 \mathrm{mV} \mathrm{s} \mathrm{s}^{-1}$ with potential range from $-0.5 \mathrm{~V}$ to $0.5 \mathrm{~V}$. The arrow indicates the progress of oxidation; (b) ATR-FTIR spectra of the AuNPs@PNE/Au electrode (blue curve), the NE/GOD/AuNPs@PNE/Au electrode (pink curve), and the PNE/GOD/AuNPs@PNE/Au electrode (red curve); (c) CV scans of the PNE/GOD/AuNPs@PNE/Au electrode at different scan rates. The inset shows the calibration curves of the electric current of the anodic and cathodic peaks; (d) Nyquist plot of EIS for the polished Au electrode (1), the PNE/GOD/Au electrode (2) and the PNE/GOD/AuNPs@PNE/Au electrode (3). The inset shows the equivalent circuit applied for the impedance data. 

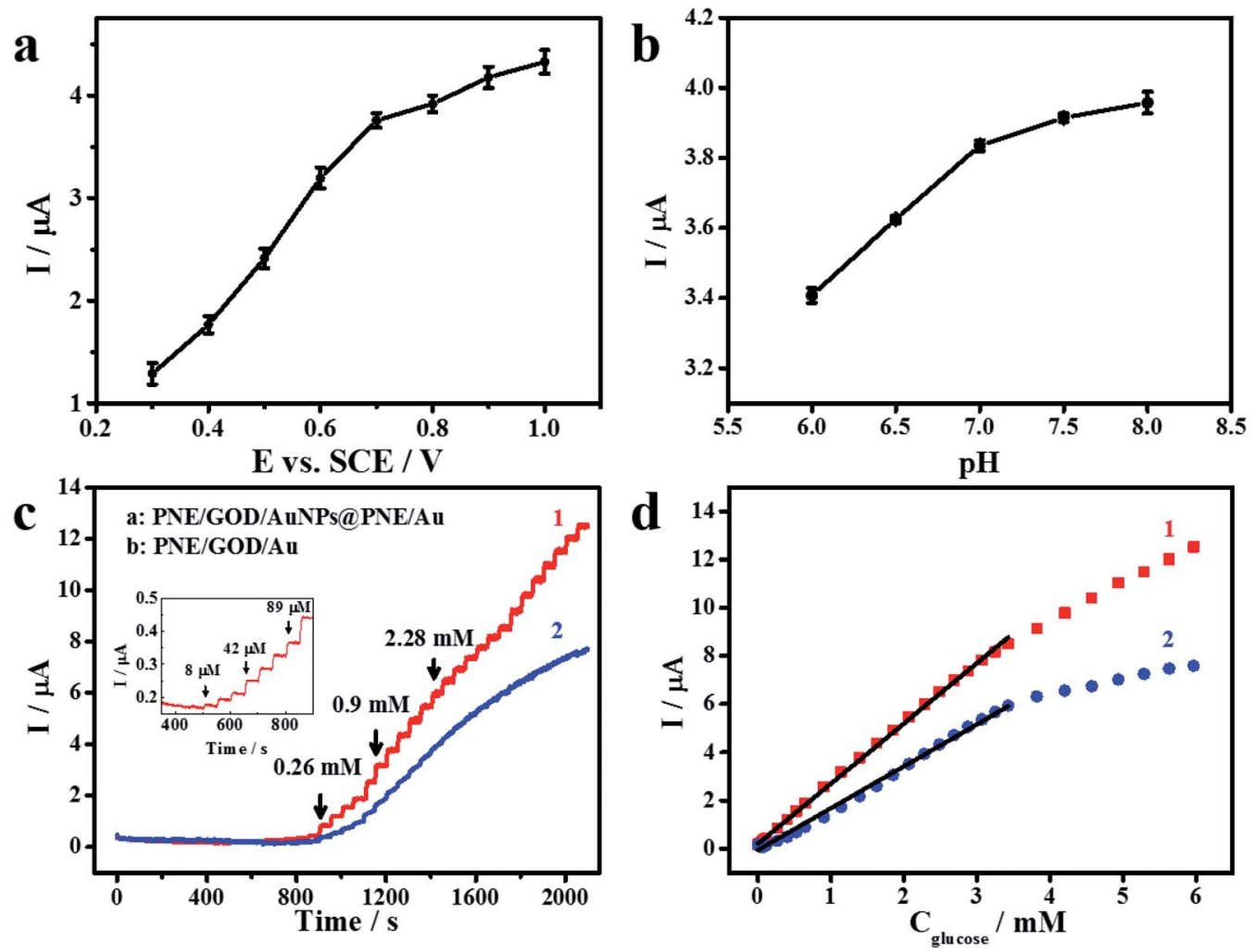

Fig. 3 (a) The static current response of the PNE/GOD/AuNPs@PNE/Au electrode to $1 \mathrm{mM}$ glucose in PBS (pH 7.4) at different potentials; (b) the static current response of the PNE/GOD/AuNPs@PNE/Au electrode to $1 \mathrm{mM}$ glucose at $0.7 \mathrm{~V}$ (vs. SCE) in PBS solutions of various pH values; (c) with successive addition of glucose, amperometic responses of the PNE/GOD/AuNPs@PNE/Au electrode (1) and the PNE/GOD/Au electrode (2) in PBS ( $\mathrm{pH} \mathrm{7.4)} \mathrm{at} \mathrm{0.7} \mathrm{V.} \mathrm{The} \mathrm{inset} \mathrm{shows} \mathrm{an} \mathrm{enlarged} \mathrm{part} \mathrm{of} \mathrm{curve} \mathrm{1;} \mathrm{(d)} \mathrm{calibration} \mathrm{curves} \mathrm{of} \mathrm{the} \mathrm{PNE/GOD/AuNPs@PNE/Au} \mathrm{electrode} \mathrm{(1)} \mathrm{and} \mathrm{the}$ $\mathrm{PNE} / \mathrm{GOD} / \mathrm{Au}$ electrode (2) according to (c).

indicating that the AuNPs@PNE nanoparticles, GOD and PNE film were successfully modified on the surface of the prepared electrodes, which inhibited the electron transfer of the $\mathrm{Fe}(\mathrm{CN})_{6}{ }^{3-/ 4-}$ redox couple.

\section{Optimization of the biosensor}

In order to obtain a high-performance glucose biosensor with a low detection limit, great sensitivity and wide detection range for glucose assays, experimental conditions, such as the detection potential and the $\mathrm{pH}$ of solution, were investigated via variation in the tested one while keeping the other fixed.

The effect of applied potentials on the response currents of the PNE/GOD/AuNPs@PNE/Au electrode was examined in PBS ( $\mathrm{pH}$ 7.4) containing $1 \mathrm{mM}$ glucose. As shown in Fig. 3a, the response current increases from $1.28 \mu \mathrm{A}$ to $4.32 \mu \mathrm{A}$ when the applied potential varies from $0.3 \mathrm{~V}$ to $1.0 \mathrm{~V}$. This indicated
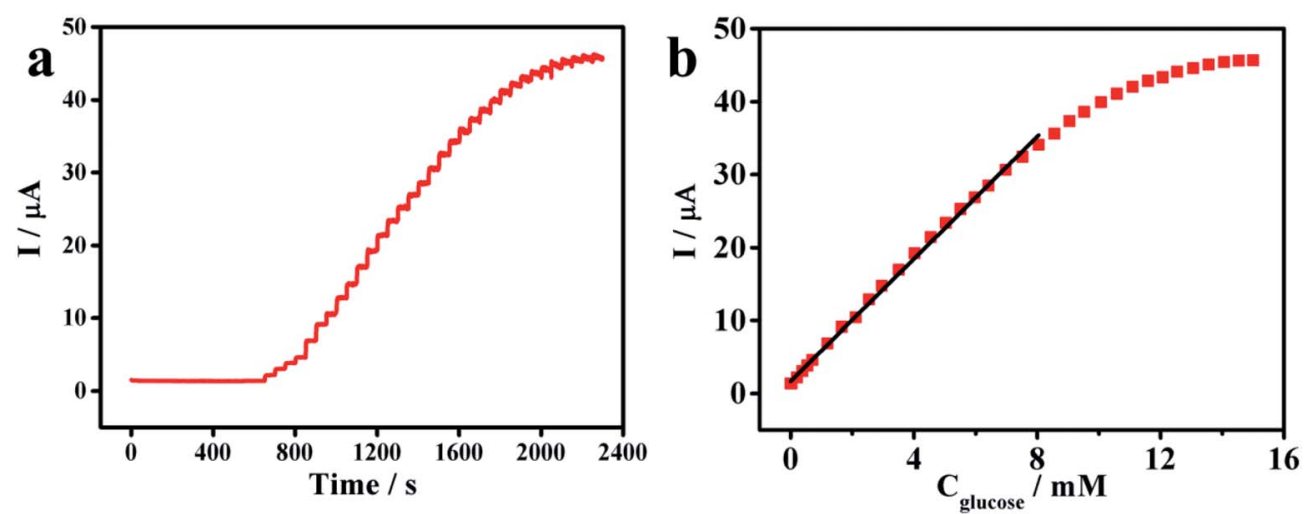

Fig. 4 (a) Amperometic responses of the PNE/GOD/AuNPs@PNE/Au electrode in PBS (pH 7.4) of $1 \mathrm{mM} B Q$ at $0.7 \mathrm{~V}$ with successive additions of glucose; (b) calibration curve of the PNE/GOD/AuNPs@PNE/Au electrode with BQ. 
Table 1 Comparison of electrochemical performances of different GOD-based electrodes

\begin{tabular}{lllll}
\hline Electrode materials & Sensitivity $\left(\mu \mathrm{Am}^{-1} \mathrm{~cm}^{-2}\right)$ & Linear range $(\mathrm{mM})$ & Limit of detection $(\mu \mathrm{M})$ & Reference \\
\hline PNE/GOD/AuNPs@PNE/Au & 35.4 & $0.003-3.43$ & 1.34 & This work \\
PNE/GOD/AuNPs@PNE/Au (with BQ) & 59.17 & $0.003-8.044$ & 1.76 & This work \\
PDA/GOD/GN & 28.4 & $0.001-4.7$ & 0.1 & 17 \\
Graphene-CdS-GOD/GCE & 1.76 & $2.0-16.0$ & 700 & 18 \\
AuNPs/GOD-MWCNTs-PVA/GCE & 16.6 & $0.5-8.0$ & 200 & 21 \\
GOD/colloidal Au/CPE & 8.4 & $0.04-0.28$ & 10 & 22 \\
GOD/chitosan/GCE & 0.233 & $0.6-2.8$ & 100 & 23
\end{tabular}

that the response of the enzyme electrode resulted from the electrochemical oxidation of hydrogen peroxide. Considering the higher current response and the fact that a high potential may result in the oxidation of many unwanted species or intermediate interferents, ${ }^{17} 0.7 \mathrm{~V}$ was chosen as the working potential in subsequent experiments. Then, the effect of the $\mathrm{pH}$ of the solution on the response current was investigated. Fig. $3 \mathrm{~b}$ shows that the response current increases slowly from $3.41 \mu \mathrm{A}$ to $3.96 \mu \mathrm{A}$ when the $\mathrm{pH}$ of the solution varies from 6.0 to 8.0 at an applied potential of $0.7 \mathrm{~V}$. Considering that the $\mathrm{pH}$ value of human blood is $7.4, \mathrm{pH} 7.4$ was selected for glucose detection.

\section{The performance of the PNE/GOD/AuNPs@PNE/Au electrode}

The amperometric response of the PNE/GOD/AuNPs@PNE/Au electrode was obtained via the successive addition of glucose in a continuously stirred $0.1 \mathrm{M}$ PBS (pH 7.4) solution at the potential of $0.7 \mathrm{~V}$, as shown in Fig. 3c. For comparison, the determination of glucose using the $\mathrm{PNE} / \mathrm{GOD} / \mathrm{Au}$ electrode was also performed. The response current of the PNE/GOD/ AuNPs@PNE/Au electrode increased rapidly with the addition of glucose and achieved over $95 \%$ of the steady-state current within $3 \mathrm{~s}$, indicating that the as-prepared modified enzyme electrode possesses excellent sensitivity and a rapid response to glucose. Furthermore, a well-defined current proportional to the glucose concentration was observed (Fig. 3d). The linear equation of the PNE/GOD/AuNPs@PNE/Au electrode in Fig. 3d is $I=2.501 C+0.201$ with $R=0.9996$. The linear range of the

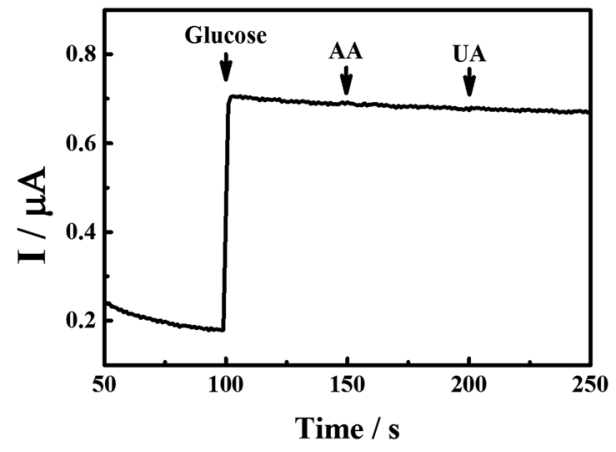

Fig. 5 Time-response current curve of the PNE/GOD/AuNPs@PNE/ Au electrode with sequential additions of $0.2 \mathrm{mM}$ glucose, $0.2 \mathrm{mM} \mathrm{AA}$, and $0.2 \mathrm{mM} \cup \mathrm{A}$ in PBS ( $\mathrm{pH} 7.4)$ at $0.7 \mathrm{~V}$. biosensor for the determination of glucose was found to be $0.003-3.43 \mathrm{mM}$ with sensitivity of $35.4 \mu \mathrm{A} \mathrm{mM} \mathrm{m}^{-1} \mathrm{~cm}^{-2}$. The detection limit of $1.34 \mu \mathrm{M}$ was estimated at a signal-to-noise ratio of 3. In contrast, the $\mathrm{PNE} / \mathrm{GOD} / \mathrm{Au}$ electrode, which showed an unstable response current, exhibited sensitivity of $25.6 \mu \mathrm{A} \mathrm{mM}^{-1} \mathrm{~cm}^{-2}$, a linear detection range of $0.089-3.43 \mathrm{mM}$ and a limit of detection of $10.29 \mu \mathrm{M}$ (signal/noise ratio $=3$ ). The higher sensitivity and lower detection limit of the PNE/GOD/ AuNPs@PNE/Au electrode demonstrated that AuNPs effectively improved the electrochemical performance of the electrode.

The dissolved $\mathrm{O}_{2}$ is a natural mediator for catalytic reaction between GOD and glucose; however, its saturated concentration in water is only about $1.3 \mathrm{mM}$. This low $\mathrm{O}_{2}$ content in solution has become a major obstacle that restricts the performance of oxidase-based biosensors. ${ }^{23}$ To further improve the electrochemical performance of the as-prepared electrode, a conventional redox mediator $p$-benzoquinone (BQ) was introduced. After the addition of $1 \mathrm{mM} \mathrm{BQ}$, the amperometric responses of the PNE/GOD/AuNPs@PNE/Au electrode to the successive injections of $1 \mathrm{mM}$ glucose are shown in Fig. $4 \mathrm{a}$. The PNE/GOD/ AuNPs@PNE/Au biosensor displayed a linear response from $0.003 \mathrm{mM}$ to $8.044 \mathrm{mM}$ with sensitivity of $59.17 \mu \mathrm{AmM}^{-1} \mathrm{~cm}^{-2}$ (Fig. 4b). The regression equation was $I=4.183 C+1.737$ with $R$ $=0.9991$ and the detection limit was $1.76 \mu \mathrm{M}$ (signal/noise ratio $=3$ ). Compared with previously reported enzyme biosensors, our PNE/GOD/AuNPs@PNE/Au electrode has an obvious advantage in sensitivity (Table 1).

\section{Michaelis-Menten constant}

Our amperometric glucose biosensor (PNE/GOD/ AuNPs@PNE/Au electrode) was based on GOD, which contains two tightly bound flavine adenine dinucleotide (FAD) cofactors; moreover, it can catalyze the electron transfer from glucose to oxygen, which is accompanied with the production of gluconolactone and hydrogen peroxide. ${ }^{24}$ The detection principle of the proposed glucose biosensor can be described as follows:

$$
\begin{gathered}
\mathrm{GOD}(\mathrm{FAD})+\text { glucose } \rightarrow \mathrm{GOD}\left(\mathrm{FADH}_{2}\right)+\text { gluconolactone } \\
\mathrm{GOD}\left(\mathrm{FADH}_{2}\right)+\mathrm{O}_{2} \rightarrow \mathrm{GOD}(\mathrm{FAD})+\mathrm{H}_{2} \mathrm{O}_{2}
\end{gathered}
$$

here, GOD(FAD) and GOD(FADH $\left.{ }_{2}\right)$ represent the oxidized and reduced forms of GOD, respectively. The quantitation of glucose 
Table 2 Determination of glucose concentration in human blood samples

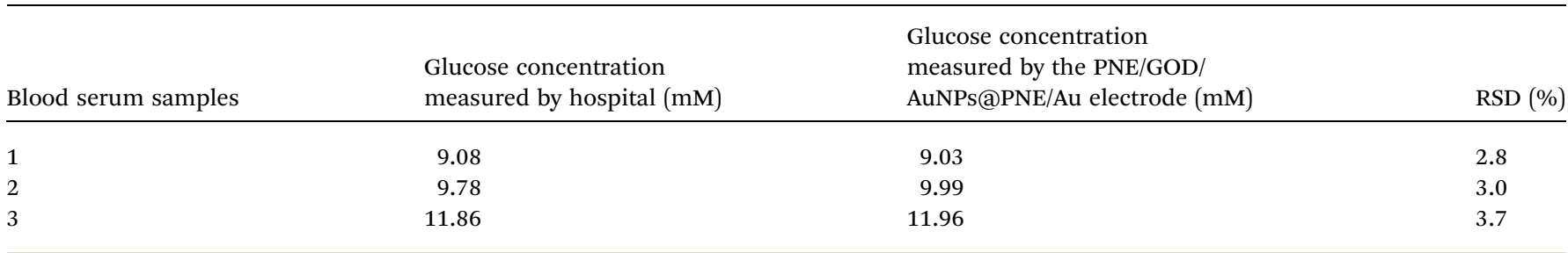

can be achieved via the electrochemical detection of the enzymatically liberated $\mathrm{H}_{2} \mathrm{O}_{2}$. Therefore, the activity of GOD on the electrode plays an important role in the performance of biosensors.

The Linweaver-Burk equation was used to calculate the apparent Michaelis-Menten constant $\left(K_{\mathrm{m}}\right)^{25}$

$$
\frac{1}{I_{\mathrm{SS}}}=\frac{1}{I_{\max }}+\frac{K_{\mathrm{m}}}{I_{\max }} \frac{1}{C}
$$

here, $I_{\mathrm{ss}}$ is the steady-state current after the addition of glucose, $I_{\max }$ is the maximum current under saturated glucose conditions and $C$ is the concentration of glucose. From the data in Fig. 3c, the $K_{\mathrm{m}}$ value of the PNE/GOD/AuNPs@PNE/Au electrode can be determined to be $6.8 \mathrm{mM}$. It is lower than the value of native GOD in solution $(33 \mathrm{mM})^{26}$ and the values reported previously for the $\mathrm{GOD} / \mathrm{GNp} / \mathrm{CS} / \mathrm{GOD} / \mathrm{GNp} / \mathrm{PAA} / \mathrm{Pt}$ modified glucose electrode $(10.5 \mathrm{mM})^{27}$ and the chitosan-gold NPmodified glassy carbon electrode $(10.1 \mathrm{mM}) \cdot{ }^{28}$ The smaller $K_{\mathrm{m}}$ value indicated that GOD immobilized by the PNE film possesses favorable enzymatic activity and satisfactory affinity for glucose.

\section{Selectivity and stability study}

Since uric acid (UA) and ascorbic acid (AA) often co-exist with glucose in serum samples, these electroactive compounds are the main interfering substances for the electrochemical detection of glucose. In order to detect the selectivity of the PNE/ GOD/AuNPs@PNE/Au electrode, the response currents to $0.2 \mathrm{mM}$ glucose, $0.2 \mathrm{mM} \mathrm{AA}$, and $0.2 \mathrm{mM}$ UA were measured at an applied potential of $0.7 \mathrm{~V}$. As shown in Fig. 5, the biosensor exhibits no response or a very weak response to AA and UA. In contrast, the large current increase after the addition of $0.2 \mathrm{mM}$ glucose was found. The results indicated that the PNE/GOD/ AuNPs@PNE/Au electrode has favorable selectivity for glucose and excellent anti-interference ability. The excellent result could be due to the specific glucose oxidation of GOD and the protection ability of the PNE film, which can effectively inhibit the penetration of negatively charged substances. The stability of the PNE/GOD/AuNPs@PNE/Au electrode was investigated at an applied potential of $0.7 \mathrm{~V}$ to compare the current responses over a period of a week. After the electrode was stored at $4{ }^{\circ} \mathrm{C}$ in PBS (pH 7.4) for a week, the response current of the PNE/GOD/ AuNPs@PNE/Au electrode in $1.0 \mathrm{mM}$ glucose was approximately $99.1 \%$ of the initial value, indicating that the biosensors have excellent stability, which can be ascribed to the excellent protection and immobilization of GOD by the PNE film.
To verify the feasibility of the clinical applications of the PNE/GOD/AuNPs@PNE/Au electrode, the biosensor was used to measure the concentrations of glucose in human blood serum. As shown in Table 2, the results obtained by the enzyme biosensor are in good agreement with those measured by the biochemical analyzer (Beckman DxC 800, USA) in the Zhongshan Hospital of the Xiamen University (Xiamen, China).

\section{Conclusions}

In summary, a PNE/GOD/AuNPs@PNE/Au electrode was successfully fabricated via a simple and green approach. In this electrode, AuNPs enhanced the electron transfer of the electrode and PNE improved the activity of the immobilized GOD. The as-prepared biosensor exhibited a low MichaelisMenten constant, a fast response to glucose and high sensitivity with a linear range of 0.003-3.43 mM. It also possessed good anti-interference ability and stability. After introducing $\mathrm{BQ}$, enhanced sensitivity $\left(59.17 \mu \mathrm{A} \mathrm{mM}{ }^{-1} \mathrm{~cm}^{-2}\right)$ and a widened linear range (0.003 to $8.044 \mathrm{mM})$ were realized. Due to these advantageous features, the biosensor can have great potentials for applications in medicine, food or other areas.

\section{Conflicts of interest}

The authors declare that they have no competing interest.

\section{Acknowledgements}

This work was supported by the National Natural Science Foundation of China (81271689, 31271009, 31870986), the Program for New Century Excellent Talents in University, the Program for New Century Excellent Talents in Fujian Province University, and the Fundamental Research Funds for the Central Universities (20720150087).

\section{References}

1 C. X. Cai and J. Chen, Anal. Biochem., 2004, 332, 75-83.

2 C. Shan, H. Yang, J. Song, D. Han, A. Ivaska and L. Niu, Anal. Chem., 2009, 81, 2378-2382.

3 C. X. Guo and C. M. Li, Phys. Chem. Chem. Phys., 2010, 12, 12153-12159.

4 Z. F. Ma and T. Ding, Nanoscale Res. Lett., 2009, 4, 1236-1240.

5 Y. Y. Horng, Y. K. Hsu, A. Ganguly, C. C. Chen, L. C. Chen and K. H. Chen, Electrochem. Commun., 2009, 11, 850-853. 
6 O. S. Muddineti, B. Ghosh and S. Biswas, Int. J. Pharm., 2015, 484, 252-267.

7 M. Rahman, L. Y. Heng, D. Futra, C. P. Chiang, Z. A. Rashid and T. Ling, Nanoscale Res. Lett., 2017, 12, 484.

8 L. Chen and S. Neethirajan, Sensors, 2015, 15, 8852-8865.

9 H. Lee, S. M. Dellatore, W. M. Miller and P. B. Messersmith, Science, 2007, 318, 426-430.

10 S. Hong, J. Kim, Y. S. Na, J. H. Park, S. J. Kim, K. Singha, G. Im, D. K. Han, W. J. Kim and H. Lee, Angew. Chem., Int. Ed., 2013, 52, 9187-9191.

11 D. Yang, X. Y. Wang, Q. H. Ai, J. F. Shi and Z. Y. Jiang, $R S C$ Adv., 2015, 5, 42461-42467.

12 R. Yuan, D. Tang, Y. Chai, X. Zhong, Y. Liu and J. Dai, Langmuir, 2004, 20, 7240-7245.

13 Y. C. Fu, P. H. Li, Q. J. Xie, X. H. Xu, L. H. Lei, C. Chen, C. Zou, W. F. Deng and S. Z. Yao, Adv. Funct. Mater., 2009, 19, 1784-1791.

14 J. Chen, R. P. Liang, X. N. Wang and J. D. Qiu, J. Chromatogr. A, 2015, 1409, 268-276.

15 J. Chen, R. P. Liang, L. L. Wu and J. D. Qiu, Electrophoresis, 2016, 37, 1676-1684.

16 S. M. Kang, S. Park, D. Kim, S. Y. Park, R. S. Ruoff and H. Lee, Adv. Funct. Mater., 2011, 21, 108-112.

17 C. J. Ruan, W. Shi, H. R. Jiang, Y. N. Sun, X. Liu, X. M. Zhang, Z. Sun, L. F. Dai and D. T. Ge, Sens. Actuators, B, 2013, 177, 826-832.
18 K. Wang, Q. Liu, Q. M. Guan, J. Wu, H. N. Li and J. J. Yan, Biosens. Bioelectron., 2011, 26, 2252-2257.

19 R. Szamocki, A. Velichko, C. Holzapfel, F. Mücklich, S. Ravaine, P. Garrigue, N. Sojic, R. Hempelmann and A. Kuhn, Anal. Chem., 2007, 79, 533-539.

20 R. Ehret, W. Baumann, M. Brischwein, A. Schwinde, K. Stegbauer and B. Wolf, Biosens. Bioelectron., 1997, 12, 29-41.

21 H. F. Zhang, Z. C. Meng, Qi. Wang and J. B. Zheng, Sens. Actuators, B, 2011, 158, 23-27.

22 S. Q. Liu and H. X. Ju, Biosens. Bioelectron., 2003, 19, 177183.

23 C. Z. Zhao, Y. Meng, C. L. Shao, L. Wan and K. Jiao, Electroanalysis, 2008, 20, 520-526.

24 X. D. Zeng, X. F. Li, L. Xing, X. Y. Liu, S. L. Luo, W. Z. Wei, B. Kong and Y. H. Li, Biosens. Bioelectron., 2009, 24, 28982903.

25 M. J. Rogers and K. G. Brandt, Biochemistry, 1971, 10, 46244630.

26 K. Yokogama and Y. Kayanuma, Anal. Chem., 1998, 70, 33683376.

27 B. Y. Wu, S. H. Hou, F. Yin, J. Li, Z. X. Zhao, J. D. Huang and Q. Chen, Biosens. Bioelectron., 2007, 22, 838-844.

28 Q. L. Sheng, Y. Shen, H. F. Zhang and J. B. Zheng, Chin. J. Chem., 2008, 26, 1244-1250. 